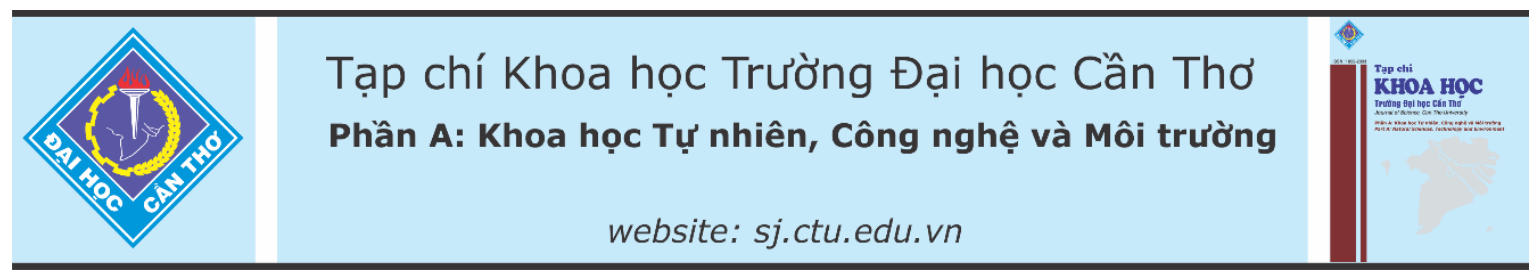

DOI:10.22144/ctu.jvn.2021.007

\title{
PHÂN LẬP MỘT SỐ HỢP CHẤT TỬ THÂN RẼ̃ NGẢI TÍM (Kaempferia parviflora WALL. EX BAKER), HỌ GỨNG (ZINGIBERACEAE)
}

\author{
Mai Đình Trị ${ }^{1 *}$, Nguyễn Tấn Phát ${ }^{1}$, Mai Thành Chí ${ }^{1}$, Phạm Quang Thọ ${ }^{1}$, Trần Huy Khiêm ${ }^{1}$, \\ Đặng Văn Sơn ${ }^{2}$ và Nguyễn Thị Ngọc Đan ${ }^{3}$ \\ ${ }^{I}$ Viện Công nghệ Hóa học - Viện Hàn lâm Khoa học và Công nghệ Việt Nam \\ ${ }^{2}$ Viện Sinh hoc Nhiệt đới - Viện Hàn lâm Khoa học và Công nghệ Việt Nam \\ ${ }^{3}$ Trung tâm Sâm và Duợc liệu thành phố Hồ Chí Minh \\ *Người chịu trách nhiệm về bài viết: Mai Đình Trị (email: maidinhtri@gmail.com)
}

\section{Thông tin chung:}

Ngày nhận bài: $28 / 12 / 2020$

Ngày nhận bài sưa: 16/01/2021

Ngày duyệt đăng: 27/03/2021

Title:

Chemical constituents of the rhizomes of Kaempferia parviflora

\section{Tù khóa:}

Kaempferia parviflora, methoxyflavone, ngải tím, thân rễ

\section{Keywords:}

Kaempferia parviflora, methoxyflavone, rhizomes

\section{ABSTRACT}

Five coumpounds including 5,7-dimethoxyflavone (1), 3,5,7trimethoxyflavone (2), di-O-methylpinocembrin (3), bisdemethoxycurcumin (4), aloe-emodin (5) were isolated from the $n$ hexane extract of Kaempferia parviflora rhizomes. Their structures were elucidated by ESI-MS, $1 D$ \& $2 D$-NMR spectra and compared their spectra with published data. Among them, compounds 4, 5 were reported for the first time from Kaempferia parviflora species.

\section{TÓM TẮT}

Năm hợp chất bao gồm 5,7-dimethoxyflavone (1), 3,5,7-trimethoxyflavone (2), di-O-methylpinocembrin (3), bisdemethoxycurcumin (4), aloe-emodin (5) được phân lập tù dịch chiết n-hexane thân rễ Ngải tím Kaempferia parviflora (ho Gừng). Cấu trúc hóa hoc các hợp chất được xác định bằng các phuơng pháp phổ nghiệm kết hợp với so sánh cấu trúc với tài liệu tham khảo. Trong đó các hơp chất phân lập 4, 5 lần đầu tìm thấy trong loài Kaempferia parviflora

\section{MỞ ĐẦU}

Cây Ngải tím có tên khoa học là Kaempferia parviflora (Zingiberaceae) là cây thân thảo lâu năm, đầu rễ thường phình lên thành dạng củ. Lá to bản, ít, phiến lá gần tròn đến dạng chỉ, có nhiều vân; cuống lá ngắn; lưỡi thường nhỏ hay không có, là mọc thành bẹ (Bùi Kim Anh và ctv., 2017). Theo truyền thống, thân rễ có màu đen đến tím được sử dụng trong thực phẩm như là một chất tạo hương vị. Thân rễ của Ngải tím trong dân gian sử dụng làm thuốc tăng cường sức khỏe, thuốc chống viêm, rối loạn tiêu hóa, ruột và tá tràng (Devi et al., 2006; Bùi Kim Anh và ctv., 2017). Các nghiên cứu gần đây cho thấy thân rễ Ngải tím có tác dụng chống sốt rét, chống virus, chống khuẩn, chống loét dạ dày và chống nấm (Yenjai el al., 2004). Trong y học cổ truyền Thái Lan, thuốc sắc của thân rễ Ngải tím dùng chữa bệnh nhiễm nấm, bất lực, dị ứng, hen, gout, tiêu chảy, kiết lị, loét dạ dày và tiểu đường (Nguyễn Phương Thảo et al., 2016). Các nghiên cứu về thành phần hóa học của loài Kaempferia parviflora cho thấy các hợp chất chủ yếu gồm flavonoid, chalcone, terpenoid, anthraquinone... Trong đó, các polymethoxyflavone được xem như là các hợp chất chính (Sea-Wong et al., 2011, Chaipech et al., 2011, 2012). Trong nghiên cứu này báo cáo kết quả về việc phân lập và xác định cấu trúc của hai hợp chất flavone, một 
flavanone, một curcuminoid và 1 anthraquinone từ thân rễ Ngãi tím là 5,7-dimethoxyflavone (1), 3,5,7trimethoxyflavone (2), di- $O$-methylpinocembrin (3), bisdemetoxycurcumin (4), aloe-emodin (5), (Hình 1).

\section{THỰC NGHIẸM}

\subsection{Thiết bị và hoá chất}

Phổ NMR được đo trên máy Bruker AM500 FTNMR (Bruker, Karlsruhe, Đức), sử dụng TMS làm chất chuẩn nội, Viện Hóa học (Viện Hàn lâm Khoa học và Công nghệ Việt Nam, Hà Nội, Việt Nam). Sắc ký lớp mỏng được thực hiện trên các tấm silica gel 60 F254 (1.05554.0001, Merck, Darmstadt, Đức) pha thường. Các vết được phát hiện bằng cách sử dụng UV ở bước sóng 254 hoặc $365 \mathrm{~nm}$ và $\mathrm{H}_{2} \mathrm{SO}_{4} 20 \% / \mathrm{EtOH}$. Sắc ký cột được thực hiện trên silica gel (240-430 mesh, Merck, Darmstadt, Đức).

\subsection{Chiết tách và phân lập}

Thân rễ Ngải tím thu hái tại tỉnh An Giang vào tháng 05 năm 2018 và được định danh bởi TS. Đặng Văn Sơn, Viện Sinh học Nhiệt Đới - Viện Hàn Lâm Khoa học và Công nghệ Việt Nam. Mẫu tiêu bản (ký hiệu KP-1539) được lưu tại Phòng Các chất có Hoạt tính Sinh học, Viện Công nghệ Hóa học. Từ 2,0 kg lượng mẫu khô thân rễ Ngải tím, tiến hành xay thành bột, chiết siêu âm với dung môi ethanol 3 lần. Toàn bộ dịch chiết cô quay áp suất kém thu được cao ethanol thô $180 \mathrm{~g}$. Thêm một lượng nước, chiết lỏng - lỏng lần lượt với các dung môi $n$-hexan, ethyl acetate (EA) thu được các cao tương ứng: cao $n$ hexan $(90 \mathrm{~g})$, EA $(20 \mathrm{~g})$ và $\mathrm{H}_{2} \mathrm{O}(60 \mathrm{~g})$.

Từ cao $n$-hexane $(90 \mathrm{~g})$ tiến hành sắc kí cột silica gel pha thường với hệ dung môi giải ly là $n$-hexane (H)-ethyl acetate $(\mathrm{EA})(100: 1,50: 1,20: 1,10: 1$ và $1: 1)$, các vết giống nhau được gom lại thành 5 phân đoạn mã hóa H1 (7.2g), H2 (20.5 g), H3 (28.4 g), H4 (17.8) và $\mathrm{H} 5$ (9.4 g). Ở phân đoạn $\mathrm{H} 2, \mathrm{H}-4$ kết quả SKBM có vết rõ nên được lựa chọn để khảo sát, các phân đoạn còn lại sẽ được nghiên cứu sau.

Từ phân đoạn $\mathrm{H} 2(20.5 \mathrm{~g})$ tiến hành sắc kí cột silica gel pha thường với hệ dung môi giải ly $n$ hexane - EA (50:1, 15:1, 5:1, 1:1) thu được 5 phân đoạn kí hiệu H2.1 (0.9 g), H2.2 (7.8 g), H2.3 (3.5 g), H2.4 (3.9 g), H2.5 (1.9 g). Ở phân đoạn H2.2 xuất hiện tủa, lọc, kết tinh lại nhiều trong dung môi $n$ hexan: acetone $(1: 1)$ thu được hợp chất $1(420 \mathrm{mg})$. Phần dịch lọc tiếp tục sắc kí cột silica gel pha thường lặp lại hệ dung môi là $\mathrm{H}$ : EA (15:1) thu được hợp chất $2(65 \mathrm{mg})$.
Ở phân đoạn H4 (17.8 g) tiến hành SKC với hệ dung môi có độ phân cực tăng dần $n$-hexane:acetone $(20: 1,10: 1,5: 1,3: 1)$ thu được 4 phân đoạn H4.1 $\rightarrow$ H4.4. Tiếp tục SKC phân đoạn H4.2 hệ dung môi $n$-hexane:acetone $(20: 1,10: 1,5: 1)$ thu được 3 phân đoạn nhỏ H4.2.1 $\rightarrow$ H4.2.3. Phân đoạn H42.1 sắc kí cột silica gel hệ dung môi $n$-hexane: acetone (15:1) thu được hợp chất $\mathbf{3}(12 \mathrm{mg})$. Hợp chất $\mathbf{4}$ (10 $\mathrm{mg}$ ) thu được từ phân đoạn $\mathrm{H} 42.2$ qua quá trình SKC silica gel hệ dung môi $n$-hexane:acetone (12:1). Cùng phân đoạn H42.3 tiếp tục sắc kí cột silica gel hệ dung môi $n$-hexane: acetone (10:1) lặp lại nhiều lần thu được hợp chất 5 (7.2 mg).

Hợp chất 1: chất bột vô định hình màu vàng nhạt: Phố ${ }^{1} \mathbf{H}$ NMR (DMSO- $d_{6}, 500 \mathrm{MHz}$ ), $\delta_{\mathrm{H}} \mathrm{ppm}$ $(\mathrm{J}, \mathrm{Hz}): 6.68(1 \mathrm{H}, \mathrm{s}, \mathrm{H}-3), 6.41(1 \mathrm{H}, \mathrm{d}, 2.0, \mathrm{H}-6)$, $6.72(1 \mathrm{H}, \mathrm{d}, 2.0, \mathrm{H}-8), 7.95(2 \mathrm{H}, \mathrm{dd}, 8.0,2.0 ; \mathrm{H}-$ 2'/H-6'), 7.49 (3H, m, H-3', H-4', H-5'), 3.79 (3H, s, $\left.5-\mathrm{OCH}_{3}\right), 3.85\left(3 \mathrm{H}, \mathrm{s}, 7-\mathrm{OCH}_{3}\right)$. Phố ${ }^{13} \mathbf{C}$ NMR (DMSO- $d_{6}, 125 \mathrm{MHz}$ ), $\delta_{\mathrm{C}}$ ppm: 159.3 (C-2), 108.1 (C-3), 175.5 (C-4), 160.1 (C-5), 96.0 (C-6), 163.6 (C-7), 93.1 (C-8), 159.0 (C-9), 108.3 (C-10), 130.8 (C-1'), 125.7 (C-2'/C-6'), 128.9 (C-3'/C-5'), 131.1 $\left(\mathrm{C}-4\right.$ '), $55.9\left(5-\mathrm{OCH}_{3}\right), 55.7\left(7-\mathrm{OCH}_{3}\right)$.

Hợp chất 2: chất bột vô định hình màu vàng nhạt: Phổ ${ }^{1} \mathbf{H}$ NMR (acetone- $d_{6}, 500 \mathrm{MHz}$ ), $\delta_{\mathrm{H}} \mathrm{ppm}$ $(\mathrm{J}, \mathrm{Hz}): 6.48(1 \mathrm{H}, \mathrm{d}, 2.0, \mathrm{H}-6), 6.74(1 \mathrm{H}, \mathrm{d}, 2.0, \mathrm{H}-$ 8), 8.01 (2H, d, 7.0, H-2'/H-6'), 7.55 (2H, d, 7.0, H3'/H-5'), 7.56 (1H, m, H-4'), 3.77 (3H, s, 3- $\left.\mathrm{OCH}_{3}\right)$, $3.86\left(3 \mathrm{H}, \mathrm{s}, 5-\mathrm{OCH}_{3}\right), 3.89\left(3 \mathrm{H}, \mathrm{s}, 7-\mathrm{OCH}_{3}\right)$. Phổ ${ }^{13} \mathrm{C}$ NMR (acetone- $\left.d_{6}, 125 \mathrm{MHz}\right), \delta_{\mathrm{C}} \mathrm{ppm}: 151.3$ (C-2), 140.8 (C-3), 171.8(C-4), 160.2 (C-5), 95.8 (C-6), 163.5 (C-7), 92.8 (C-8), 157.9 (C-9), 108.5 (C-10), 129.6 (C-1'), 127.4 (C-2'/C-6'), 128.1 (C3'/C-5'), 125.5 (C-4'), $55.6\left(5-\mathrm{OCH}_{3}\right), 55.8$ (5$\left.\mathrm{OCH}_{3}\right), 59.1\left(7-\mathrm{OCH}_{3}\right)$.

Hợp chất 3: chất bột vô định hình màu trắng ngà: Phổ ${ }^{1} \mathbf{H}$ NMR (acetone- $d_{6}, 500 \mathrm{MHz}$ ), $\delta_{\mathrm{H}} \mathrm{ppm}$ $(\mathrm{J}, \mathrm{Hz}): 5.47(1 \mathrm{H}, \mathrm{dd} 12.5 ; 3.0 ; \mathrm{H}-2), 2.65(1 \mathrm{H}, \mathrm{dd}$ $16.5 ; 3.0 ; \mathrm{H}-3 \mathrm{a}), 2.96(1 \mathrm{H}$, dd 16.5; 12.5; H-3b), 6.19 (2H, s, H-6, H-8), 8.05 (2H, m, H-2'/H-6'), 7.45 (2H, m, H-3'/H-5'), 7.42 (1H, m, H-4'), 3.82 $\left(3 \mathrm{H}, \mathrm{s}, 5-\mathrm{OCH}_{3}\right), 3.86\left(3 \mathrm{H}, \mathrm{s}, 7-\mathrm{OCH}_{3}\right)$. Phổ ${ }^{13} \mathbf{C}$ NMR (acetone- $\left.d_{6}, 125 \mathrm{MHz}\right), \delta_{\mathrm{C}} \mathrm{ppm}: 79.8(\mathrm{C}-2)$, 46.3 (C-3), 187.7 (C-4), 165.6 (C-5), 94.4 (C-6), 166.6 (C-7), 93.6 (C-8), 163.2 (C-9), 106.7 (C-10), 140.5 (C-1'), 129.4 (C-2'/C-6'), 127.1 (C-3'/C-5'), $129.1\left(\mathrm{C}-4\right.$ '), $56.0\left(5-\mathrm{OCH}_{3}\right), 56.1\left(7-\mathrm{OCH}_{3}\right)$.

Hợp chất 4: chất bột vô định hình màu vàng: Phổ ${ }^{1} \mathbf{H}$ NMR (acetone-d $\left.\boldsymbol{\sigma}, 500 \mathrm{MHz}\right), \boldsymbol{\delta}_{\mathrm{H}} \mathrm{ppm}(\mathrm{J}$, Hz): 5.98 (1H, s; H-2), $6.82(2 \mathrm{H}$; d; 16.0, H-3, H3'), $7.62(2 \mathrm{H}$; d; 17.0, H-4, H-4'), 7.57 (4H, d, 
8.5Hz, H-6/H-6', H-10/H-10'), 6.91 (4H; d; 8.5 Hz; H-7/H-7'; H-9/H-9'). Phổ ${ }^{13}$ C NMR (acetone- $d_{6}$, $125 \mathrm{MHz}), \delta_{\mathrm{C}} \mathrm{ppm}: 101.6(\mathrm{C}-1), 184.3$ (C-2/2'), 122.0 (C-3/3'), 141.0 (C-4/4'), 127.7 (C-5/5'), 130.9 (C-6/6', C-10/10'), 116.7 (C-7/7', C-9/9'), 160.5 (C-8/8').

Hợp chất 5: chất bột vô định hình màu vàng: Phổ ${ }^{1} \mathrm{H}-\mathrm{NMR}$ (acetone- $\left.d_{6}, 500 \mathrm{MHz}\right), \delta_{\mathrm{H}} \mathrm{ppm}(\mathrm{J}$,<smiles></smiles>

1: $\mathrm{R}_{1}=\mathrm{R}_{2}=-\mathrm{OCH}_{3}, \mathrm{R}_{3}=\mathrm{H}$

2: $\mathrm{R}_{1}=\mathrm{R}_{2}=\mathrm{R}_{3}=-\mathrm{OCH}_{3}$<smiles>COC(=C\C(=O)/C=C/c1ccc(O)cc1)/C=C/c1ccc(O)cc1</smiles>

4
Hz): 7.82 (1H; m; H-6); $7.81(1 \mathrm{H} ; \mathrm{d} ; 1.5 ; \mathrm{H}-4) ; 7.80$ $(1 \mathrm{H} ; \mathrm{m} ; \mathrm{H}-5) ; 7.36(1 \mathrm{H} ; \mathrm{m} ; \mathrm{H}-7) ; 7.33(1 \mathrm{H} ; \mathrm{d} ; 2.0$; $\mathrm{H}-2) ; 12.0(1 \mathrm{H}, \mathrm{s} ; 1-\mathrm{OH}), 11.9(1 \mathrm{H}, \mathrm{s} ; 8-\mathrm{OH}), 4.80$ $(2 \mathrm{H}$; d; 5.5, H-11), $4.54(1 \mathrm{H} ; \mathrm{t}, 4.5 ; 11-\mathrm{OH}) . \mathbf{P h}$ ${ }^{13} \mathrm{C}$ NMR (acetone- $d_{6}, 125 \mathrm{MHz}$ ), $\delta_{\mathrm{C}}$ ppm: 163.9 (C-1), 125.2 (C-2), 155.0 (C-3), 118.5 (C-4), 120.4 (C-5), 138.3 (C-6), 121.8 (C-7), 163.5 (C-8), 194.0 (C-9), 182.3 (C-10), 117.1 (C-1a), 134.8 (C-4a), 135.0 (C-5a), 115.6 (C-8a), 64.0 (C-11).<smiles>COc1cc(OC)c2c(c1)O[C@H](c1ccccc1)CC2=O</smiles><smiles></smiles>

5

Hình 1. Cấu trúc của các hợp chất 1-5 từ thân rễ ngải tím

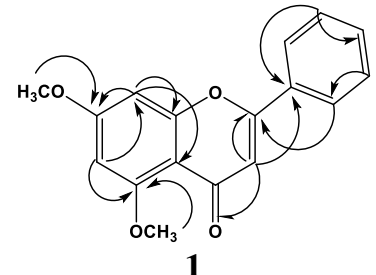

1

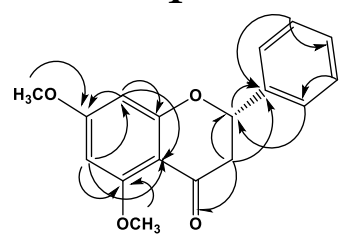

3

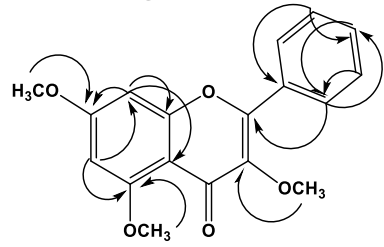

2

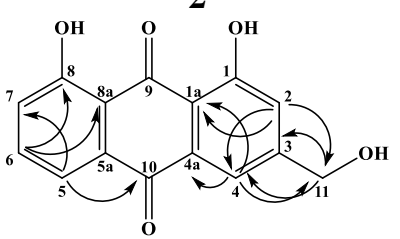

5

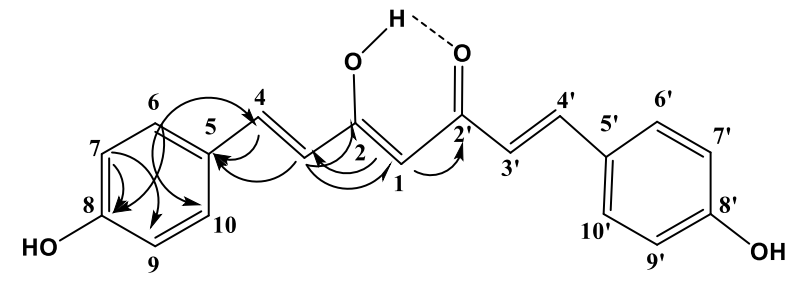

4

Hình 2. Tương tác HMBC hợp chất 1-5 từ thân rễ ngải tím

\section{KÊT QUẢ VÀ THẢO LUẬN}

Hợp chất 1 được phân lập dưới dạng bột vô định hình màu vàng. Phổ khối lượng ESI-MS của hợp chất 1 xuất hiện các pic ion phân tử giả ở $m / z: 283.28$ $[\mathrm{M}+\mathrm{H}]^{+}, m / z: 305.22[\mathrm{M}+\mathrm{Na}]^{+}$, phù hợp công thức phân tử của 1 là $\mathrm{C}_{17} \mathrm{H}_{14} \mathrm{O}_{4}$. 
Phổ ${ }^{1}$ H-NMR ( $\left.500 \mathrm{MHz}, \mathrm{DMSO}-d_{6}, \delta \mathrm{ppm}\right)$ của 1 cho các tín hiệu cộng hưởng của 2 proton methine vòng thơm ghép cặp meta ở $\delta_{\mathrm{H}} 6.41(1 \mathrm{H}, \mathrm{d}, J=2.0$ $\mathrm{Hz}, \mathrm{H}-6)$ và $6.72(1 \mathrm{H}, \mathrm{d}, J=2.5 \mathrm{~Hz}, \mathrm{H}-8), 5$ proton methine vòng thơm ở $\delta_{\mathrm{H}} 7.95(2 \mathrm{H}, \mathrm{dd}, J=8.0$ và 2.0 $\mathrm{Hz}, \mathrm{H}-2^{\prime}, \mathrm{H}-6$ '), $\delta_{\mathrm{H}} 7.49$ (3H, m, H-3', H-4', H-5'), cho thấy chất $\mathbf{1}$ có hai vòng thơm, một vòng mang một nhóm thế và một vòng mang bốn nhóm thế. Ngoài ra, còn có sự hiện diện 6 proton của hai nhóm methoxyl ở $\delta_{\mathrm{H}} 3.79\left(3 \mathrm{H}, s, 5-\mathrm{OCH}_{3}\right)$ và $3.85(3 \mathrm{H}, s$, $\left.7-\mathrm{OCH}_{3}\right), 1$ proton olefine ở $\delta_{\mathrm{H}} 6.68(1 \mathrm{H}, s, \mathrm{H}-3)$. Phổ ${ }^{13}$ C-NMR $\left(125 \mathrm{MHz}, \mathrm{DMSO}-d_{6}, \delta \mathrm{ppm}\right)$ kết hợp với phổ DEPT, phổ HSQC của $\mathbf{1}$ cho các tín hiệu cộng hưởng của 17 carbon, trong đó có 1 carbon carbonyl ở $\delta_{\mathrm{C}} 175.5(\mathrm{C}-4), 4$ carbon tứ cấp của vòng thơm mang oxy ở $\delta_{\mathrm{C}} 163.6(\mathrm{C}-7), \delta_{\mathrm{C}} 160.1$ $(\mathrm{C}-5), \delta_{\mathrm{C}} 159.3(\mathrm{C}-2), \delta_{\mathrm{C}} 159.0(\mathrm{C}-9), 2$ carbon tứ cấp của vòng thơm ở $\delta_{\mathrm{C}} 130.8\left(\mathrm{C}-1^{\prime}\right)$ và $\delta_{\mathrm{C}} 108.3(\mathrm{C}$ 10), 8 carbon methine ở $\delta_{\mathrm{C}} 108.1(\mathrm{C}-3), \delta_{\mathrm{C}} 96.0$ (C6), $\delta_{\mathrm{C}} 93.1(\mathrm{C}-8), \delta_{\mathrm{C}} 125.7\left(\mathrm{C}-2^{\prime}, \mathrm{C}-6^{\prime}\right), \delta_{\mathrm{C}} 128.9(\mathrm{C}-$ 3 ', C-5'), $\delta_{\mathrm{C}} 131.1\left(\mathrm{C}-4^{\prime}\right)$ và 2 carbon methoxyl ở $\delta_{\mathrm{C}}$ $55.9\left(5-\mathrm{OCH}_{3}\right), 55.7\left(7-\mathrm{OCH}_{3}\right)$, dự đoán 1 là flavone mang 2 nhóm thế methoxyl.

Phổ HMBC của 1 cho thấy 2 proton ghép cặp meta $\delta_{\mathrm{H}} 6.41(1 \mathrm{H}, \mathrm{d}, J=2.0 \mathrm{~Hz}, \mathrm{H}-6)$ và $6.73(1 \mathrm{H}$, $\mathrm{d}, J=2.0 \mathrm{~Hz}, \mathrm{H}-8)$ đều cho tương quan với carbon tứ cấp của vòng thơm mang oxy ở $\delta_{\mathrm{C}} 163.6$ là $\mathrm{C}-7$, $\delta_{\mathrm{C}} 160.1$ là $\mathrm{C}-5$ và $\delta_{\mathrm{C}} 159.0$ là $\mathrm{C}-9$ và carbon tứ cấp ở $\delta_{\mathrm{C}} 108.3$ là $\mathrm{C}-10$. Mặt khác, proton của hai nhóm methoxyl ở $\delta_{\mathrm{H}} 3.79\left(3 \mathrm{H}, s, 5-\mathrm{OCH}_{3}\right)$ và $3.85(3 \mathrm{H}, s$, $7-\mathrm{OCH}_{3}$ ) lần lượt cho tương quan với 2 carbon tứ cấp của vòng thơm mang oxy ở $\delta_{\mathrm{C}} 160.1(\mathrm{C}-5)$ và $\delta_{\mathrm{C}}$ $163.6(\mathrm{C}-7)$ xác nhận 2 nhóm methoxyl vào $\mathrm{C}-5$ và C-7 khung flavone. Phổ HMBC còn cho thấy H-3', H-4', H-5' tương quan với carbon tại $\delta_{\mathrm{C}} 125.7$ (C-2', C-6'), $\delta_{\mathrm{C}} 128.9$ (C-3', C-5'), $\delta_{\mathrm{C}} 131.1$ (C-4') và $\delta_{\mathrm{C}}$ 130.8 nên $\delta_{C} 130.8$ là C-1'. Và H-2', H-6' tương quan với $\delta_{\mathrm{C}} 130.8\left(\mathrm{C}-1^{\prime}\right), \delta_{\mathrm{C}} 131.1\left(\mathrm{C}-4^{\prime}\right)$ và $\delta_{\mathrm{C}} 159.3$ nên carbon ở $\delta_{\mathrm{C}} 159.3$ chính là $\mathrm{C}-2$. Từ các dữ liệu phổ trên và so sánh với tài liệu tham khảo (Sutthanut et al., 2007) xác định cấu trúc của 1 là 5,7dimethoxyflavone

Họ̣p chất 2 thu được có dạng bột vô định hình màu vàng nhạt.

Phổ ${ }^{1}$ H-NMR và ${ }^{13}$ C-NMR của 2 gần giống hợp chất $\mathbf{1}$, tuy nhiên trên vòng $\mathrm{C}$ của hợp chất $\mathbf{2}$ biến mất một proton ở [ $\delta_{\mathrm{H}} 6.68(1 \mathrm{H}, s, \mathrm{H}-3) / \delta_{\mathrm{C}} 108.1(\mathrm{C}-$ $3)$ ], thay vào đó xuất hiện carbon tứ cấp ở [ $\delta_{\mathrm{C}} 140.8$, $\mathrm{C}-3]$ và một nhóm methoxyl tại $\delta_{\mathrm{C}} 55.6\left(3-\mathrm{OCH}_{3}\right)$. Như vậy hợp chất 2 cũng là một flavone mang 03 ba nhóm methoxyl. Trên phổ HMBC cho thấy các nhóm methoxyl được nối vào $C-3, C-5$ và $C-7$ do có sự tương quan của proton này với carbon của chúng. Từ các dữ liệu phổ NMR và so sánh tài liệu (Sutthanut et al., 2007) hợp chất 2 xác định là 3,5,7-trimethoxyflavone

\section{Hợp chất 3 thu được có dạng bột trắng ngà.}

Phổ ${ }^{1} \mathbf{H}-\mathbf{N M R}\left(500 \mathrm{MHz}\right.$, acetone- $\left.d_{6}, \delta \mathrm{ppm}\right)$ của hợp chất 3 cho thấy ở vùng trường thấp xuất hiện tín hiệu 7 proton methine thơm $\left[\delta_{\mathrm{H}} 6.19(2 \mathrm{H} ; \mathrm{s} ; \mathrm{H}-6, \mathrm{H}-\right.$ 8)], [ $\left.\delta_{\mathrm{H}} 7.56\left(2 \mathrm{H} ; \mathrm{m} ; \mathrm{H}-2^{\prime} ; \mathrm{H}-6^{\prime}\right)\right] ; 7.45(2 \mathrm{H} ; \mathrm{m} ; \mathrm{H}-$ $\left.3^{\prime} ; \mathrm{H}-5^{\prime}\right)$ ] và $\left.7.42\left(1 \mathrm{H} ; \mathrm{m} ; \mathrm{H}-4^{\prime}\right)\right]$. Ở vùng trường cao có sự xuất hiện tín hiệu của 1 proton oxymethine $\left[\delta_{\mathrm{H}}\right.$ $5.51(1 \mathrm{H} ; \mathrm{dd} ; \mathrm{J}=12.5 ; 3.0 \mathrm{~Hz} ; \mathrm{H}-2)]$ và 2 proton methylene $\left[\delta_{\mathrm{H}} 2.69(1 \mathrm{H} ; \mathrm{dd} ; \mathrm{J}=16.5 ; 3.0 \mathrm{~Hz} ; \mathrm{H}-3 \mathrm{a})\right]$ và $\left[\delta_{\mathrm{H}} 2.96(1 \mathrm{H} ; \mathrm{dd} ; \mathrm{J}=16.5 ; 12.5 \mathrm{~Hz} ; \mathrm{H}-3 \mathrm{~b})\right]$, cùng tín hiệu của 2 nhóm methoxyl $\left[\delta_{\mathrm{H}} 3.82(3 \mathrm{H} ; \mathrm{s} ; 5-\right.$ $\mathrm{OMe})]$ và $\left[\delta_{\mathrm{H}} 3.86(3 \mathrm{H} ; \mathrm{s} ; 7-\mathrm{OMe})\right]$. Phổ ${ }^{13} \mathbf{C}-\mathbf{N M R}$ $\left(125 \mathrm{MHz}\right.$, acetone- $\left.d_{6}, \delta \mathrm{ppm}\right)$ của hợp chất 3 cho thấy có tín hiệu của 17 carbon. Trong đó có 1 carbon carbonyl nhóm ketone $\left[\delta_{\mathrm{C}} 187.7\right.$; C-4]; 3 carbon thơm gắn với oxy [ $\left.\delta_{\mathrm{C}} 163.2 ; \mathrm{C}-9\right],\left[\delta_{\mathrm{C}} 165.6 ; \mathrm{C}-5\right]$, [ $\left.\delta_{\mathrm{C}} 166.6 ; \mathrm{C}-7\right]$; 2 carbon thơm bậc 4 [ $\delta_{\mathrm{C}} 106.7$; C$10],\left[\delta_{\mathrm{C}} 140.5 ; \mathrm{C}-1{ }^{\prime}\right] ; 7$ carbon methine thơm $\left[\delta_{\mathrm{C}}\right.$

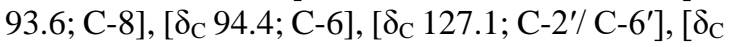
$\left.129.4 ; \mathrm{C}-3^{\prime} / \mathrm{C}-5^{\prime}\right], \quad\left[\delta_{\mathrm{C}} 129.1 ; \mathrm{C}-4^{\prime}\right] ; 1$ carbon oxymethine $\left[\delta_{\mathrm{C}} 79.8 ; \mathrm{C}-2\right] ; 1$ carbon methylene $\left[\delta_{\mathrm{C}}\right.$ $46.3 ; \mathrm{C}-3]$ và 2 nhóm methoxyl $\left[\delta_{\mathrm{C}} 56.0 ;(5-\mathrm{OMe})\right.$, 56.1 (7-OMe) ]. Phân tích dữ liệu phổ trên cho thấy hợp chất 3 có cấu trúc của một flavanone.

Phân tích phổ hai chiều HMBC của hợp chất 3 cho thấy 2 proton ở $\delta_{\mathrm{H}} 6.19(2 \mathrm{H}, \mathrm{s})$ đều cho tương quan với carbon thơm gắn oxy ở $\delta_{\mathrm{C}} 166.6(\mathrm{C}-7), \delta_{\mathrm{C}}$ $165.6(\mathrm{C}-5), \delta_{\mathrm{C}} 163.2(\mathrm{C}-9)$ và carbon vòng thơm không mang oxy ở $\delta_{\mathrm{C}} 106.7$ (C-10). Như vậy hai carbon ở $\delta_{\mathrm{C}} 94.4$ và 93.6 chính là $\mathrm{C}-6$ và $\mathrm{C}-8$. Mặt khác, trên phổ HMBC còn cho thấy xuất hiện tín hiệu 6 proton của hai nhóm methoxyl ở $\delta_{\mathrm{H}} 3.82(3 \mathrm{H}$, $\left.\mathrm{s}, 5-\mathrm{OCH}_{3}\right)$ và $3.86\left(3 \mathrm{H}, \mathrm{s}, 7-\mathrm{OCH}_{3}\right)$ lần lượt cho tương quan với 2 carbon vòng thơm mang oxy ở $\delta_{\mathrm{C}}$ 165.6 (C-5) và $\delta_{C} 166.6(\mathrm{C}-7)$ giúp xác nhận 2 nhóm methoxyl gắn vào $\mathrm{C}-5, \mathrm{C}-7$. Ngoài ra proton ở 7.45 $\left.\left(2 \mathrm{H} ; \mathrm{m} ; \mathrm{H}-3^{\prime} ; \mathrm{H}-5^{\prime}\right)\right]$ cùng cho tương quan HMBC với $\delta_{\mathrm{C}} 129.4\left(\mathrm{C}-2^{\prime}, \mathrm{C}-6^{\prime}\right)$ và carbon tứ cấp ở $\delta_{\mathrm{C}} 140.5$ nên carbon này chính là $\mathrm{C}-1$ '. Tương tác $\mathrm{HMBC}$ cũng cho thấy tín hiệu proton ở $7.56(2 \mathrm{H} ; \mathrm{m} ; \mathrm{H}-$ $\left.2^{\prime} / 6^{\prime}\right)$ ]; lại cho tương quan với carbon ở $\delta_{\mathrm{C}} 129.4(\mathrm{C}-$ 4 ') và $\delta_{\mathrm{C}} 79.4$, do đó carbon ở $\delta_{\mathrm{C}} 79.4$ chính là $\mathrm{C}-2$ và vòng $\mathrm{B}$ sẽ gắn vào khung tại vị trí $\mathrm{C}-2$, proton $\left[\delta_{\mathrm{H}}\right.$ $2.69(1 \mathrm{H} ; \mathrm{dd} ; \mathrm{J}=16.5 ; 3.0 \mathrm{~Hz} ; \mathrm{H}-3 \mathrm{a})]$ và $\left[\delta_{\mathrm{H}} 2.96\right.$ $(1 \mathrm{H} ; \mathrm{dd} ; \mathrm{J}=16.5 ; 12.5 \mathrm{~Hz} ; \mathrm{H}-3 \mathrm{~b})]$ cùng cho tương quan với $\delta_{\mathrm{C}} 140.5\left(\mathrm{C}-1^{\prime}\right), \delta_{\mathrm{C}} 79.4(\mathrm{C}-2)$ và carbon carbonyl ở $\delta_{\mathrm{C}} 187.7(\mathrm{C}-4)$ nên proton methylene chính là $\mathrm{H}-3$ và proton oxymethine là $\mathrm{H}-2$. Mặt khác 
phân tích hằng số ghép spin của proton oxymethine [ $\left.\delta_{\mathrm{H}} 5.51(1 \mathrm{H} ; \mathrm{dd} ; \mathrm{J}=12.5 ; 3.0 \mathrm{~Hz} ; \mathrm{H}-2)\right]$ cho thấy $\mathbf{J}$ $=12.5 \mathrm{~Hz}$ chứng tỏ $\mathrm{H}-2$ ghép trục-trục với $\mathrm{H}-3 \mathrm{~b}$, nhu vậy $\mathrm{H}-2$ định hướng ở vị trí $\beta$, vòng phenyl định hướng $\alpha$. Tiến hành so sánh dữ liệu phổ của hợp chất 3 với hợp chất di- $O$-methylpinocembrin cho thấy có sự tương hợp (Chavi et al., 2009). Vậy cấu trúc hợp chất 3 là di- $O$-methylpinocembrin. vàng.

Họ̣p chất 4 thu được chất bột vô định hình màu

Phổ ${ }^{1} \mathbf{H}$ NMR $\left(500 \mathrm{MHz}\right.$, acetone- $\left.d_{6}, \delta \mathrm{ppm}\right)$ xuất hiện tín hiệu của hai vòng benzen thế 1,4$\delta_{\mathrm{H}}[7.57$ (4H, d, 8.5Hz, H-6/H-6', H-10/H-10'); 6.91 (4H; d; 8.5 Hz; H-7/H-7';H-9/H-9')]; hai nối đôi cấu hình trans $\delta_{\mathrm{H}}[7.62(2 \mathrm{H} ; \mathrm{d} ; 17.0 \mathrm{~Hz} ; \mathrm{H}-4 / \mathrm{H}-4$ '); 6.82 (2H; d; 16.0 Hz; H-3/H-3'); một proton của nhóm methine $\delta_{\mathrm{H}} 5.98(1 \mathrm{H}, \mathrm{s}, \mathrm{H}-1)$. Phổ ${ }^{13} \mathbf{C}$ NMR (125 $\mathrm{MHz}$, acetone- $\left.d_{6}, \delta \mathrm{ppm}\right)$ cho thấy hợp chất $\mathbf{4}$ có 19 carbon bao gồm 6 carbon bậc bốn, trong đó hai carbonyl $\delta_{\mathrm{C}} 184.3$ (C-2/C-2'), hai carbon vòng thơm mang oxy $\delta_{\mathrm{C}} 160.5$ (C-8/C-8'), 13 carbon bậc ba $\delta_{\mathrm{C}}$ [122.0 (C-3/C-3); 141.0 (C-4/C-4'); 130.9 (C-6/C6', C-10/C-10'); 116.7 (C-7/C-7', C-9/C-9'); 101.6 (C-1)]. Việc các tín hiệu cộng hưởng xuất hiện trùng lặp với cường độ gấp đôi trên phổ ${ }^{1} \mathrm{H} N M R$ và ${ }^{13} \mathrm{C}$ NMR cho biết hợp chất 4 có cấu trúc đối xứng.

Phổ HMBC cho thấy hai tín hiệu proton tại $\delta_{\mathrm{H}}$ [7.57 $(4 \mathrm{H} ; \mathrm{d} ; 8.5) ; 6.91(4 \mathrm{H} ; \mathrm{d} ; 8.5)]$ cùng tương quan với một carbon bậc bốn mang oxy $\delta_{\mathrm{C}} 160.5$ (C8/C-8') nên nhóm hydroxyl gắn trên carbon này (C8/C-8'). Carbon bậc bốn còn lại của vòng benzene được xác định nhờ vào tương quan giữa tín hiệu $\delta_{\mathrm{H}}$ 6.91 với carbon bậc bốn $\delta_{\mathrm{C}} 127.7$ (C-5/C-5'). Hai proton cấu hình trans ở $\left[\delta_{\mathrm{H}} 6.82(2 \mathrm{H} ; \mathrm{d} ; 16.0 \mathrm{~Hz})\right.$ và $\delta_{\mathrm{H}} 7.62(2 \mathrm{H} ; \mathrm{d} ; 17.0 \mathrm{~Hz})$ cùng cho tương quan với carbon bậc bốn vòng thơm ở $\delta_{\mathrm{C}} 127.7$ (C-5/C-5')], đồng thời các proton $\delta_{\mathrm{H}} 7.62$ của vòng thơm tương quan với carbon mang nối đôi ngoài vòng $\delta_{\mathrm{C}} 141.0$ (C-4/C-4'). Tương quan HMBC giữa H-6/H-6' với carbon olefine bậc ba $\delta_{\mathrm{C}} 141.0$ chứng tỏ này là $\mathrm{C}$ 4/C-4', do đó tín hiệu tại $\delta_{C} 122.0$ là của C-3/C-3'. Trong phổ HMBC, các proton $\mathrm{H}-3 / \mathrm{H}-3$ ' và $\mathrm{H}-4 / \mathrm{H}-$ 4' cùng tương quan với carbon carbonyl ở $\delta_{\mathrm{C}} 184.3$ khẳng định nhóm carbonyl gắn trực tiếp vào nối đôi. Tín hiệu cộng hưởng của proton nối đôi xuất hiện dưới dạng singlet $\delta_{\mathrm{H}} 5.98(1 \mathrm{H}, \mathrm{s})$ cho tương quan với carbon carbonyl và carbon C-3 của nối đôi. Những tín hiệu proton $\delta_{\mathrm{H}} 5.98(1 \mathrm{H}, \mathrm{s})$ và carbon olefin bậc ba $\delta_{\mathrm{C}} 101.6$ đặc trưng của các hợp chất curcuminoid tồn tại dưới dạng hổ biến ketone-enol. Từ các nhận định phổ nghiệm nêu trên, kết hợp tài liệu tham khảo
(Jayaprakasha et al., 2006). hợp chất 4 được xác định là bisdemethoxycurcumin. Đây là hợp chất lần đầu tìm thấy trong loài.

Họ̣p chất 5 thu được chất bột, màu vàng.

Phổ ${ }^{1}$ H-NMR (500 MHz, acetone- $d_{6}$, ) cho thấy sự hiện diện 5 proton vòng thơm ở $\delta_{\mathrm{H}} 7.82(1 \mathrm{H} ; \mathrm{m}$; H-6); $7.81(1 \mathrm{H} ; \mathrm{d} ; \mathrm{J}=1.5 ; \mathrm{H}-4) ; 7.80(1 \mathrm{H} ; \mathrm{m} ; \mathrm{H}-5)$; $7.36(1 \mathrm{H} ; \mathrm{m} ; \mathrm{H}-7) ; 7.33(1 \mathrm{H} ; \mathrm{d} ; \mathrm{J}=2.0 ; \mathrm{H}-2) ; 2$ tín hiệu singlet của 2 proton nhóm $-\mathrm{OH}$ kiềm nối tại $\delta_{\mathrm{H}}$ $12.0(1 \mathrm{H}, \mathrm{s} ; 1-\mathrm{OH}), \delta_{\mathrm{H}} 11.9(1 \mathrm{H}, \mathrm{s} ; 8-\mathrm{OH}), 2$ proton nhóm oxymethylene tại $\delta_{\mathrm{H}} 4.80(2 \mathrm{H} ; \mathrm{d} ; \mathrm{J}=5.5 \mathrm{~Hz})$. Ngoài ra còn có 1 proton nhóm $\mathrm{OH}$ ở $\delta_{\mathrm{H}} 4.54(1 \mathrm{H}$; t, J=4.5 Hz ). Phố ${ }^{13} \mathbf{C}-\mathbf{N M R}(125 \mathrm{MHz}$, acetone$d_{6}$,) kết hợp với phổ DEPT cho các tín hiệu đặc trưng của khung anthraquinone 14 carbon gồm hai carbon tứ cấp của vòng thơm mang oxygene $\left[\delta_{\mathrm{C}} 163.9\right.$ và $163.5]$, năm carbon tứ cấp vòng thơm $\left[\delta_{C} 155.0\right.$; $135.0 ; 134.8 ; 117.1 ; 115.6]$, năm carbon methine vòng thơm $\left[\delta_{\mathrm{C}} 138.3 ; 125.2 ; 121.8 ; 120.4 ; 118.5\right]$, hai carbon carbonyl $\left[\delta_{C} 194.0 ; 182.3\right]$. Ngoài ra còn có một carbon oxymethylene ở [ $\left.\delta_{\mathrm{C}} 64.0\right]$.

Ở vùng từ trường thấp cho thấy carbon carbonyl ở [ $\left.\delta_{C} 194.0\right]$ và $\left[\delta_{C} 182.3\right]$ gần 2 nhóm $\mathrm{OH}$ kiềm nối là $\mathrm{C}-9$ và $\mathrm{C}-10$. Trên phổ $\mathrm{HMBC}$ proton ở $\delta_{\mathrm{H}} 7.80$ $(1 \mathrm{H} ; \mathrm{m} ; \mathrm{H}-5)$ cùng cho các tương quan với carbon carbonyl $\left[\delta_{\mathrm{C}} 182.3 ; \mathrm{C}-10\right]$ và 2 carbon tứ cấp ở $\left[\delta_{\mathrm{C}}\right.$ 135.0 và 115.6$]$, ngoài ra proton thơm tại $\delta_{\mathrm{H}} 7.82$ (1H; m; H-6) lại cho tương quan với carbon tứ cấp mang oxy ở [ $\left.\delta_{\mathrm{C}} 163.5\right]$ và carbon bậc 4 tại $\left[\delta_{\mathrm{C}} 115.6\right]$ như vậy hai carbon bậc 4 lần lượt tại $\left[\delta_{C} 115.6\right]$ là C$8 \mathrm{a}$ và $\left[\delta_{\mathrm{C}} 135.0\right]$ là $\mathrm{C}-5 \mathrm{a}$, carbon tại $\left[\delta_{\mathrm{C}} 163.5\right]$ chính là $\mathrm{C}-8$. Ngoài ra proton ở $7.33(1 \mathrm{H} ; \mathrm{d} ; \mathrm{J}=2.0 ; \mathrm{H}-2)$ cùng cho các tương quan $\mathrm{HMBC}$ với carbon carbon tứ cấp ở [ $\left.\delta_{\mathrm{C}} 117.1\right]$ và carbon methine ở [ $\left.\delta_{\mathrm{C}} 118.5\right]$, proton ở $\delta_{\mathrm{H}} 7.81(1 \mathrm{H} ; \mathrm{d} ; \mathrm{J}=1.5 ; \mathrm{H}-4)$ lại cho tương quan với carbon tứ cấp mang oxy tại $\left[\dot{\delta}_{\mathrm{C}} 163.9\right]$ và carbon bậc 4 tại [ $\left.\delta_{C} 134.8\right]$ như vậy hai carbon bậc 4 tại $\left[\delta_{\mathrm{C}} 117.1\right.$ và 134.8$]$ lần lượt là $\mathrm{C}-1 \mathrm{a}$ và $\mathrm{C}-4 \mathrm{a}$, carbon tại $\left[\delta_{\mathrm{C}} 163.9\right]$ chính là $\mathrm{C}-1$. Proton nhóm oxymethylene ngoài tương quan với $\mathrm{C}-2$ và $\mathrm{C}-4$ còn tương quan với carbon ở $\delta_{\mathrm{C}} 155.0$ nên carbon này chính là $\mathrm{C}-3$, do đó nhóm oxymethylen sẽ nối vào C-3. Từ dữ liệu phổ so sánh với tài liệu (Danielsen et al., 1992), hợp chất 5 được xác định là aloeemodin. Đây là hợp chất lần đầu phân lập từ trong loài.

\section{KẾT LUẬN}

Bằng kỹ thuật sắc ký cột trên silica gel pha thường kết hợp với sắc ký bản mỏng với các hệ dung môi giải ly khác nhau, năm hợp chất tinh khiết được phân lập từ phân đoạn cao $n$-hexane của thân rễ Ngải 
tím. Cấu trúc được xác định bằng phổ cộng hưởng từ hạt nhân kết hợp với so sánh tài liệu tham khảo, các hợp chất được xác định là 5,7-dimethoxyflavone (1) 3,5,7-trimethoxyflavone (2), di-Omethylpinocembrin (3), bisdemethoxycurcumin (4), aloe-emodin (5). Trong đó hợp chất $\mathbf{4}$ và 5 lần đâu tìm thấy trong loài.

\section{LỜI CẢM ƠN}

Công trình được tài trợ bởi Viện Hàn lâm Khoa học và Công nghệ Việt Nam trong đề tài mã số NCVCC15.02/20-20.

\section{TÀI LIẾU THAM KHẢO}

Anh, B.D., Tiên, D.D., Điền, P.G., Hùng, P.D., Dương, P.Q., Hoàng, V.D. (2017). Bước đầu nghiên cứu thành phần hóa học và hoạt tính sinh học thân rễ Ngải đen. Tạp chí Duoọc liệu, Viện Dươc Liệu, 22(1), 24 - 29.

Chaipech, S., Morikawa, T., Ninomiya, K., Yoshikawa, M., Pongpiriyadacha, Y., Hayakawa, T., \& Muraoka, O. (2012). Structures of two new phenolic glycosides, kaempferiaosides A and B, and hepatoprotective constituents from the rhizomes of Kaempferia parviflora. Chemical and Pharmaceutical Bulletin, 60(1), 62-69.

Chaipech, S., Morikawa, T., Ninomiya, K., Yoshikawa, M., Pongpiriyadacha, Y., Hayakawa, T., \& Muraoka, O. (2012). New flav-3-en-3-ol glycosides, kaempferiaosides $\mathrm{C}$ and $\mathrm{D}$, and acetophenone glycosides, kaempferiaosides $\mathrm{E}$ and F, from the rhizomes of Kaempferia parviflora. Journal of natural medicines, 66(3), 486-492.

Chavi Y., Suchana W., Siripit P and Bungon S. (2009). Structural modification of 5,7dimethoxyflavone from Kaempferia parviflora and biological activities. Archives of Pharmacal Research, 32 (9), 1179-1184.

Danielsen, K., Aksnes, D. W., \& Francis, G. W. (1992). NMR study of some anthraquinones from rhubarb. Magnetic resonance in chemistry, 30(4), 359-360.

Devi, N. B., Das, A. K \& Singh, P. K. (2016). Kaempferia parviflora (Zingiberaceae): A new record in the flora of Manipur. Int J Innov Sci Eng Technol, 3, 661-5

Jayaprakasha, G. K., Ohnishi-Kameyama, M., Ono, H., Yoshida, M., \& Jaganmohan Rao, L. (2006). Phenolic constituents in the fruits of Cinnamomum zeylanicum and their antioxidant activity. Journal of agricultural and food chemistry, 54(5), 1672-1679.

Sae-Wong, C., Matsuda, H., Tewtrakul, S., Tansakul, P., Nakamura, S., Nomura, Y., \& Yoshikawa, M. (2011). Suppressive effects of methoxyflavonoids isolated from Kaempferia parviflora on inducible nitric oxide synthase (iNOS) expression in RAW 264.7 cells. Journal of ethnopharmacology, 136(3), 488-495.

Sutthanut K., Sripanidkulchai. B., Yenjai C and Jay M. (2007). Simultaneous identification and quantitation of 11 flavonoid constituents in Kaempferia parviflora by gas chromatography. Journal of Chromatography A. 1143, 227-233.

Thao, N. P., Luyen, B. T. T., Kim, J. H., Jo, A. R., Yang, S. Y., Dat, N. T., ... \& Kim, Y. H. (2016). Soluble epoxide hydrolase inhibitory activity by rhizomes of Kaempferia parviflora Wall. ex Baker. Medicinal Chemistry Research, 25(4), 704-711.

Yenjai, C., Prasanphen, K., Daodee, S., Wongpanich, V., \& Kittakoop, P. (2004). Bioactive flavonoids from Kaempferia parviflora. Fitoterapia, 75(1), 89-92. 